\title{
Direction finding with a single spatially stretched vector sensor in the presence of mutual coupling
}

\author{
Ting Shu ${ }^{1 *}$ (D), Kun Wang ${ }^{1}$, Jin $\mathrm{He}^{1}$ and Zhong Liu
}

\begin{abstract}
This paper is concerned with DOA estimation using a single-electromagnetic vector sensor in the presence of mutual coupling. Firstly, we apply the temporally smoothing technique to improve the identifiability limit of a single-vector sensor. In particular, we establish sufficient conditions for constructing temporally smoothed matrices to resolve $K>2$ incompletely polarized (IP) monochromatic signals with a single-vector sensor. Then, we propose an efficient ESPRIT-based method, which does not require any calibration signals or iterative operations, to jointly estimate the azimuth-elevation angles and the mutual coupling coefficients. Finally, we derive the Cramér-Rao bound (CRB) for the problem under consideration.
\end{abstract}

Keywords: Electromagnetic vector sensor, Direction finding, ESPRIT, Mutual coupling

\section{Introduction}

Direction finding using a single-electromagnetic vector sensor (EMVS) has played an important role in applications such as radar, wireless communications and seismic exploration. An EMVS consists of six components, three identical but orthogonally oriented electrically short dipoles, and another three identical but orthogonally oriented magnetically small loops. An EMVS can therefore measure all the six electromagnetic field components induced by any electromagnetic incidence. After Li [1], and Nehorai and Paldi [2] first introduced the EMVS measurement model to the signal processing community, a variety of studies regarding signal processing with a single EMVS [2-8] have been extensively carried out. These methods ignore the mutual coupling across the six antenna component, which ultimately destroys the underlying model assumptions needed for their efficient implementations. Consequently, ignoring this mutual coupling effect can seriously degrade the performance the above mentioned algorithms. Therefore, it is of

\footnotetext{
*Correspondence: tingshu@sjtu.edu.cn

'Shanghai Key Laboratory of Intelligent Sensing and Recognition, Department of Electronic Engineering, Shanghai Jiaotong University, Shanghai 200240, People's Republic of China

Full list of author information is available at the end of the article
}

great significance to develop algorithms for simultaneous mutual coupling calibration and parameter estimation.

In the last few years, many advanced array calibration methods have been reported. These algorithms include the maximum likelihood algorithm [9], the iterative autocalibration method [10], the auxiliary sensor-based methods [11-14], the cumulant-based method [15], the Rankreduction (RARE)-based calibration methods $[16,17]$, the sparse representation-based methods [18-20], and the matrix reconstruction method [21]. However, some of these methods require a set of calibration signals/auxiliary sensors $[9,11-14]$ or iterative/high order statistics/nonlinear optimization computations [10, 15-20]. Moreover, all such methods are designed for scalar sensor arrays and are not applicable to the vector sensor arrays. Calibration of mutual coupling for vector sensors has been studied recently in [22] and [23]. These two methods can offer closed-form solutions for coupling matrix and parameter estimation. However, they require a coupling-free auxiliary vector sensor and design of a reference signal.

The aforementioned scalar sensor array calibration methods have been a strong motivation for us to develop new joint calibration and estimation methods for vector sensor arrays, and the contribution of the work lies in that direction. The proposed method is outlined as follows:

\section{Springer Open}

(c) The Author(s). 2018 Open Access This article is distributed under the terms of the Creative Commons Attribution 4.0 International License (http://creativecommons.org/licenses/by/4.0/), which permits unrestricted use, distribution, and reproduction in any medium, provided you give appropriate credit to the original author(s) and the source, provide a link to the Creative Commons license, and indicate if changes were made. 
the temporal smoothing technique is firstly applied to improve the identifiability limit of a single vector. In particular, sufficient conditions for constructing temporally smoothed matrices to resolve $K$ incompletely polarized (IP) monochromatic signals with a single-vector sensor are established. An ESPRIT-based method is then developed for jointly estimating the azimuth-elevation angles and the mutual coupling coefficients. This method does not require any calibration signals or iterative operations. The Cramér-Rao bound (CRB) for the problem under consideration is also derived.

Throughout the paper, scalar quantities are denoted by lowercase letters. Lowercase bold type faces are used for vectors and uppercase letters for matrices. Superscripts $T$, $H, *$, and $\dagger$ represent the transpose, conjugate transpose, complex conjugate and pseudo inverse, respectively, while $\otimes$ and $\odot$, respectively, symbolize the Kronecker-product operator and the Khatri-Rao (column-wise Kronecker) matrix product. $\mathbf{I}_{m}$ and $\mathbf{0}_{m, n}$, respectively, stand for the $m \times m$ identity matrix and $m \times n$ zero matrix.

\section{Mathematical data model and assumptions}

Assume that there are $K$ uncorrelated monochromatic transverse electromagnetic signals, parameterized by $\left\{\theta_{1}, \phi_{1}\right\}, \cdots,\left\{\theta_{K}, \phi_{K}\right\}$, after having traveled through a homogeneous isotropic medium, impinge upon a single spatially stretched EMVS. The EMVS consists of three orthogonal electric dipoles and another three orthogonal magnetic loops, with the dipole triad being located at
$(0,0)$ and the loop triad being located at $\left(d_{x}, d_{y}\right)$, as shown in Fig. 1. The parameter $0 \leq \theta_{k}<\pi$ denotes the elevation angle of the $k$ th signal, and $0 \leq \phi_{k}<2 \pi$ represents the azimuth angle. The $6 \times 1$ data vector measured by the EMVS at time instant $t$ can be expressed as [2]

$$
\mathbf{z}(t)=\sum_{k=1}^{K} \mathbf{A}_{k} \mathbf{s}_{k}(t)+\mathbf{n}(t)=\mathbf{A} \mathbf{s}(t)+\mathbf{n}(t)
$$

where

$$
\begin{gathered}
\mathbf{A}_{k}=\mathbf{A}\left(\theta_{k}, \phi_{k}\right)=\left[\mathbf{a}_{1, k}, \mathbf{a}_{2, k}\right]=\left[\begin{array}{cc}
\mathbf{v}_{1, k} & \mathbf{v}_{2, k} \\
q_{k} \mathbf{v}_{2, k} & -q_{k} \mathbf{v}_{1, k}
\end{array}\right] \\
\mathbf{v}_{1, k}=\left[\cos \theta_{k} \cos \phi_{k}, \cos \theta_{k} \sin \phi_{k},-\sin \theta_{k}\right]^{T} \\
\mathbf{v}_{2, k}=\left[-\sin \phi_{k}, \cos \phi_{k}, 0\right]^{T} \\
q_{k}=e^{j 2 \pi / \lambda\left(d_{x} \sin \theta_{k} \cos \phi_{k}+d_{y} \sin \theta_{k} \sin \phi_{k}\right)} \\
\mathbf{A}=\left[\mathbf{A}_{1}, \cdots, \mathbf{A}_{K}\right] \\
\mathbf{s}_{k}(t)=\left[s_{k, 1}(t), s_{k, 2}(t)\right]^{T} \\
=\left[\beta_{k, 1} e^{j\left(\omega_{k, 1} t+\psi_{k, 1}\right)}, \beta_{k, 2} e^{j\left(\omega_{k, 2} t+\psi_{k, 2}\right)}\right]^{T} \\
\mathbf{s}(t)=\left[\mathbf{s}_{1}^{T}(t), \cdots, \mathbf{s}_{K}^{T}(t)\right]^{T} \\
\mathbf{n}(t)=\left[n_{1}(t), \cdots, n_{6}(t)\right]^{T}
\end{gathered}
$$

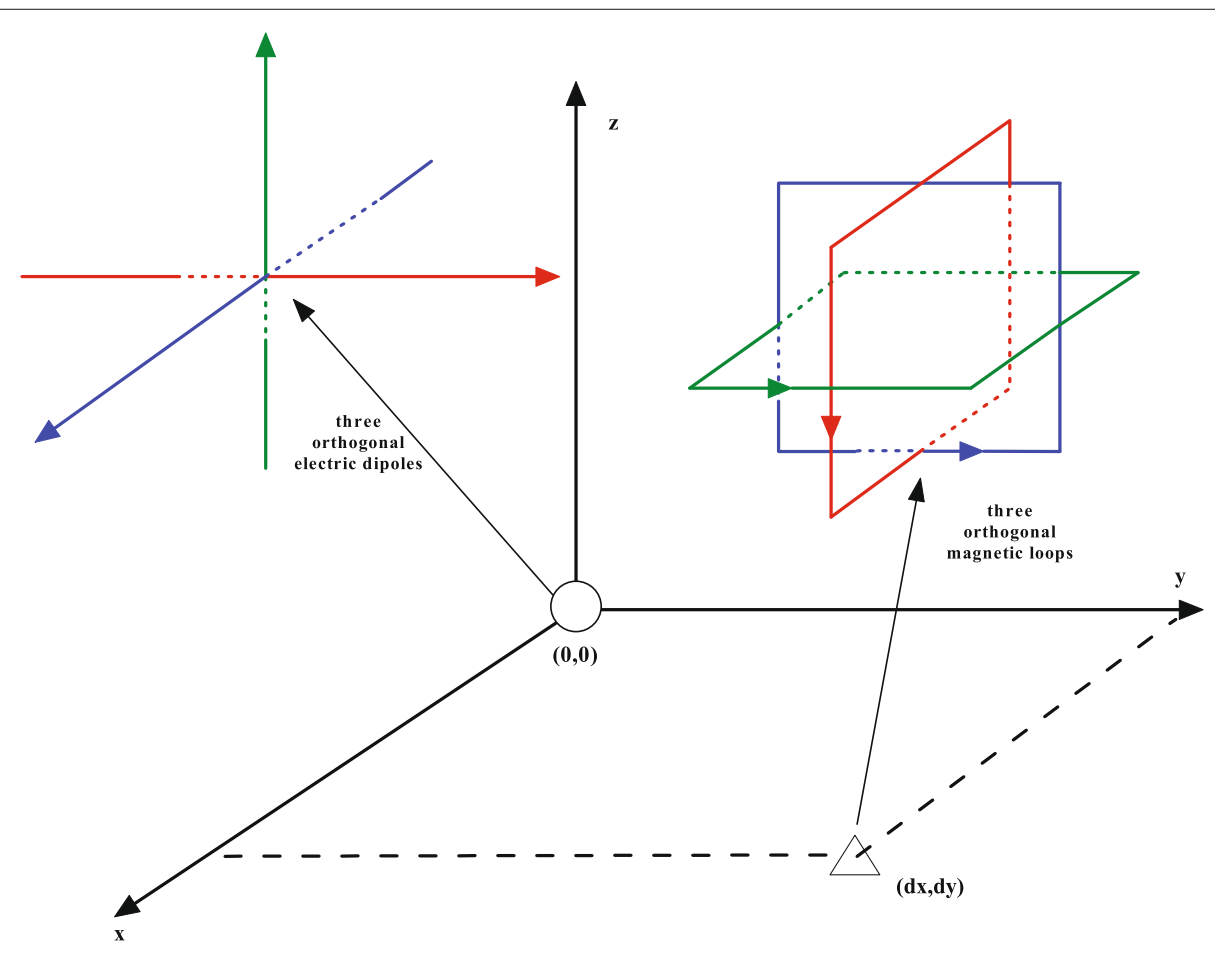

Fig. 1 Illustration of a spatially stretched EMVS 
In the above equations,

- $\mathbf{A}_{k}$ is the $6 \times 2$ EMVS response of the $k$ th electromagnetic signal.

- $\mathrm{A}$ is the $6 \times 2 K$ EMVS steering matrix.

- $\mathbf{s}_{k}(t)$ is a $2 \times 1$ vector, representing the two entries of the $k$ th transmitted signal.

- In (7), $\beta_{k, i}, \omega_{k, i}$ and $\psi_{k, i}, i,=1,2$, respectively, represent the energy, frequency, and the uniformly distributed random phase of the $i$ th entry of the $k$ th signal.

- $\mathbf{n}(t)$ is the $6 \times 1$ noise vector.

Note that the rank of the covariance matrix of the $k$ th signal $\mathbf{R}_{\mathbf{s}_{k}}=E\left\{\mathbf{s}_{k}(t) \mathbf{s}_{k}^{H}(t)\right\}$ is related to the polarization state of the $k$ th signal $[24,25]$. For the case of an incompletely polarized (IP) signal, the covariance matrix $\mathbf{R}_{s_{k}}$ is of rank-2, whereas for the case of completely polarized (CP) signal, $\mathbf{R}_{s_{k}}$ becomes rank deficient. In other words, the IP signal possesses two spatial degrees of freedom, whereas the CP signal has only one. Referring back to (7), if $\omega_{k, 1} \neq \omega_{k, 2}$, then the signal is IP, and its polarization varies with time. On the other hand, if $\omega_{k, 1}=\omega_{k, 2}=\omega_{k}$, then the signal is $\mathrm{CP}$, and it has constant polarization. For the CP signal, $\mathbf{s}_{k}(t)$ can be expressed as [25]

$$
\mathbf{s}_{k}(t)=\mathbf{g}_{k} s_{k}(t)=\left[\begin{array}{c}
\sin \gamma_{k} e^{j \eta_{k}} \\
\cos \gamma_{k}
\end{array}\right] s_{k}(t)
$$

where $\gamma_{k}$ and $\eta_{k}$ are polarization parameters referred to as the auxiliary polarization angle and polarization phase difference, respectively, and $s_{k}(t)=\beta_{k} e^{j\left(\omega_{k} t+\psi_{k}\right)}$ is the $k$ th transmitted signal. Thus, for the case of $K C P$ signals, the data vector in (1) reduces to the one used in [3].

The data vector model in (1) is only valid for ideal vector sensors. For practical vector sensors, the mutual coupling across the six vector sensor components is not negligible. In such a realistic situation, the signal received by a vector sensor component is no longer related to the wavefield incident on that component only, but can be expressed as a linear combination of the wavefields incident onto all the six vector sensor components. To take the coupling effect into account, the data model in (1) needs to be modified by including a $6 \times 6$ matrix term $M$, referred to as the coupling matrix, which expresses the aforementioned linear combination. Therefore, the data vector in the presence of mutual coupling can be written as

$$
\mathbf{z}(t)=\mathbf{M A s}(t)+\mathbf{n}(t)
$$

The mutual coupling matrix $\mathbf{M}$ may contain 36 distinct parameters. Thus, a full coupling matrix model usually requires too many parameters to be estimated. Therefore, a simplified version of $\mathbf{M}$ is required. If a priori knowledge on the mutual coupling matrix is available, then its exploitation can be useful in reducing the number of parameters to be estimated, thus, making the estimation procedure simpler. In light of this consideration, we assume that the displacement between the dipole triad and the loop triad is large enough so that the mutual coupling coefficients between the dipole triad and the loop triad are zero. This hypothesis can be justified by the fact that the coupling decreases quite rapidly with distance [11]. Furthermore, since the components in the dipole triad and loop triad are orthogonal to one another, all the components of a vector sensor would experience the same coupling effect. Therefore, the mutual coupling matrix $\mathbf{M}$ can be formulated as

$$
\mathbf{M}=\mathbf{I}_{2} \otimes \mathbf{C}=\mathbf{I}_{2} \otimes\left[\begin{array}{lll}
c_{1} & c_{2} & c_{2} \\
c_{2} & c_{1} & c_{2} \\
c_{2} & c_{2} & c_{1}
\end{array}\right]
$$

where $c_{1}$ and $c_{2}$ represent the self and mutual coupling coefficients, respectively.

With a total of $N$ snapshots taken at the distinct instants $\left\{n \Delta_{T}: n=1, \cdots, N\right\}$, the problem is to determine the DOA's $\left\{\left(\theta_{k}, \phi_{k}\right), k=1, \cdots, K\right\}$ of the $K$ signals and the coupling matrix $\mathbf{C}$ from these snapshots. The following assumptions are made:

1. The parameters $\left(\theta_{1}, \phi_{1}\right), \cdots,\left(\theta_{K}, \phi_{K}\right)$ are pairwise distinct.

2. The value of $K$ is known or correctly estimated.

3. The coupling coefficients $c_{1} \neq c_{2}$ so that the coupling matrix $\mathbf{C}$ is non-singular.

4. The impinging signals are IP and are uncorrelated with one another. This implies that the frequencies $\omega_{1,1} \neq \omega_{1,2} \neq \cdots \neq \omega_{K, 1} \neq \omega_{K, 2}$.

5. The noise is zero-mean, complex Gaussian, and is statistically independent of all the signals.

\section{Joint angle and mutual coupling matrix estimation}

\subsection{Temporal smoothing}

The authors in [4] have found that the maximum number of arbitrary electromagnetic sources uniquely identifiable by one vector sensor is two. That is, the data matrix in (11) is rank deficient if the number of incoming signals is greater than two. In this subsection, we will apply the temporal smoothing technique [26] to deal with this rank deficiency problem. We will also show that under certain conditions, the temporal smoothing technique can restore the rank of the data matrix.

Define a $6 \times N$ data matrix $\mathbf{Z}=\left[\mathbf{z}\left(\Delta_{T}\right), \cdots, \mathbf{z}\left(N \Delta_{T}\right)\right]$, where $\mathbf{z}\left(\Delta_{T}\right), \mathbf{z}\left(2 \Delta_{T}\right), \cdots, \mathbf{z}\left(N \Delta_{T}\right)$ are the $N$ snapshots sampled at time instants $\Delta_{T}, 2 \Delta_{T}, \cdots, N \Delta_{T}$, respectively. For simplicity of analysis, we will neglect the noise terms. Then, we define $P$ temporally shifted data subsets of $\mathbf{Z}$, where each contains $N-P-1$ data samples. The first and the $p$ th temporally shifted data subsets can be expressed as 

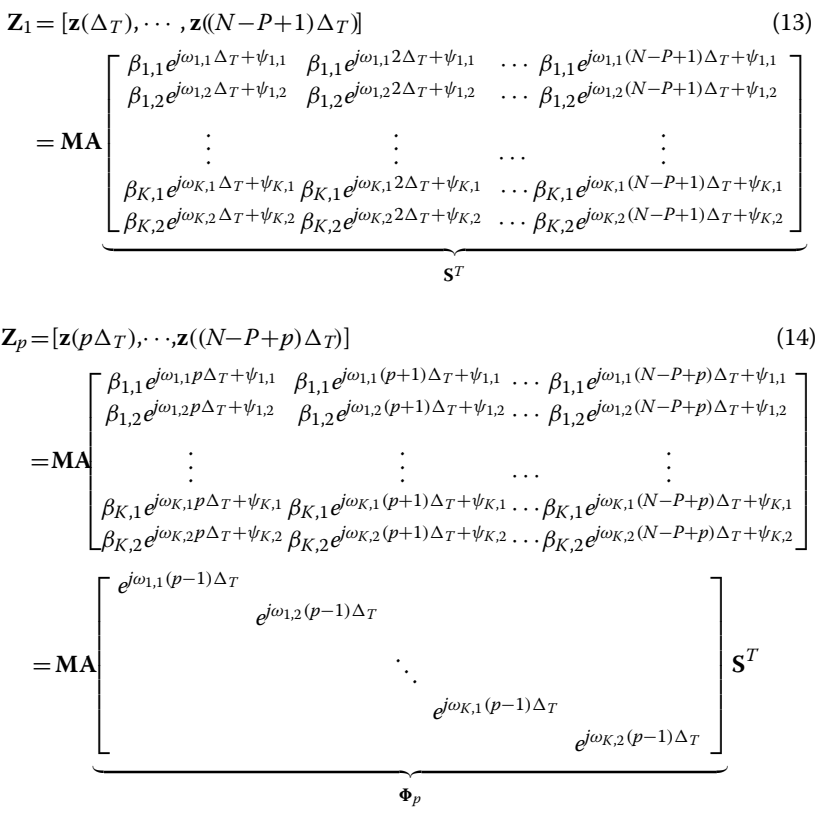

where

$$
\begin{array}{r}
\boldsymbol{\Phi}_{p}=\operatorname{diag}\left[e^{j \omega_{1,1}(p-1) \Delta_{T}}, e^{j \omega_{1,2}(p-1) \Delta_{T}}, \ldots,\right. \\
\left.e^{j \omega_{K, 1}(p-1) \Delta_{T}}, e^{j \omega_{K, 2}(p-1) \Delta_{T}}\right]
\end{array}
$$

is a diagonal matrix that is only dependent on the time delay and the frequencies of the signals, and

$$
\mathbf{S}=\left[\mathbf{s}\left(\Delta_{T}\right), \cdots, \mathbf{s}\left((N-P+1) \Delta_{T}\right)\right]^{T}
$$

is a $(N-P+1) \times 2 K$ signal matrix. Then, for $p=1, \cdots, P$, we will have $P$ different data sets $\left\{\mathbf{Z}_{1}, \cdots, \mathbf{Z}_{P}\right\}$. Note that these $P$ data sets differ from one another in view of the fact the the matrices $\boldsymbol{\Phi}_{p}$ differ from one set to another. Next, the $6 P \times(N-P+1)$ temporally smoothed data matrix is defined by stacking $\mathbf{Z}_{p}$ for $p=1, \cdots, P$ as

$$
\mathbf{Z}_{\mathrm{TS}}=\left[\mathbf{Z}_{1}^{T}, \cdots, \mathbf{Z}_{P}^{T}\right]^{T}
$$

Theorem 1 If $P \geq 2 K$ and $N \geq 4 K-1$, then the temporally smoothed data matrix $\boldsymbol{Z}_{T S}$ is of full rank $2 K$.

Proof The matrix $\mathbf{Z}_{\mathrm{TS}}$ can be expressed in a columnwise Kronecker matrix product form as

$$
\mathbf{Z}_{\mathrm{TS}}=(\boldsymbol{\Psi} \odot \mathbf{M A}) \mathbf{S}^{T}
$$

where

$\boldsymbol{\Psi}=\left[\begin{array}{ccccc}1 & 1 & \cdots & 1 & 1 \\ e^{j \omega_{1,1} \Delta_{T}} & e^{j \omega_{1,2} \Delta_{T}} & \cdots & e^{j \omega_{K, 1} \Delta_{T}} & e^{j \omega_{K, 2} \Delta_{T}} \\ \vdots & \vdots & \cdots & \vdots & \vdots \\ e^{j \omega_{1,1}(P-1) \Delta_{T}} & e^{j \omega_{1,2}(P-1) \Delta_{T}} & \cdots & e^{j \omega_{K, 1}(P-1) \Delta_{T}} & e^{j \omega_{K, 2}(P-1) \Delta_{T}}\end{array}\right]$

Since all the signals are assumed to be IP and have distinct frequencies, the Vandermonde matrix $\mathbf{S}$ is of full column rank $2 K$ if and only if $(N-P+1) \geq 2 K$. Next, by results in [27], we have

$$
\operatorname{rank}(\boldsymbol{\Psi} \odot \mathbf{M A}) \leq \min \{2 K, \operatorname{rank}(\boldsymbol{\Psi}) \cdot \operatorname{rank}(\mathbf{M A})\}
$$

and a sufficient condition for equality is to have $\boldsymbol{\Psi}$ and/or MA tall and full rank. Then, if $P \geq 2 K$, the Vandermonde matrix $\Psi$ is tall and is of rank $2 K$. In this case,

$$
\operatorname{rank}(\boldsymbol{\Psi} \odot \mathbf{M A})=\min \{2 K, 2 K \cdot \operatorname{rank}(\mathbf{M A})\}=2 K
$$

Finally, combining $P \geq 2 K$ with $(N-P+1) \geq 2 K$, we have $\mathbf{Z}_{\text {TS }}$ to be of full rank $2 K$, if $P \geq 2 K$ and $N \geq 4 K-1$, since ( $\boldsymbol{\Psi} \odot \mathbf{M A})$ is of full column rank and $\mathbf{S}^{T}$ is of full row rank. This concludes the proof.

Theorem 1 establishes sufficient but not necessary conditions for constructing temporally smoothed matrices to resolve $K$ IP monochromatic signals with a single-vector sensor. Specially, on the basis of Theorem 1, an infinite number of uncorrelated signals with distinct frequencies may potentially be resolved as $N$ approaches infinity.

\subsection{Angle and mutual coupling matrix estimation}

In this subsection, we propose an ESPRIT-based algorithm to estimate the angles and the coupling matrix from the data matrix $\mathbf{Z}_{\mathrm{TS}}$. For analytical purposes, we consider the ideal noiseless case. Let $\mathbf{E}_{s}$ be the $6 P \times 2 K$ signal-subspace eigenvector matrix, whose columns are the $6 P \times 1$ signal-subspace eigenvectors associated with the $2 K$ largest eigenvalues of $\mathbf{Z}_{\mathrm{TS}} \mathbf{Z}_{\mathrm{TS}}^{H}$. Using the basic idea of ESPRIT [28], we have

$$
\mathbf{E}_{s}=(\mathbf{\Psi} \odot \mathbf{M A}) \mathbf{T}=\mathbf{B} \mathbf{T}
$$

where $\mathbf{B}=\boldsymbol{\Psi} \odot \mathbf{M A}$, and $\mathbf{T}$ is a unique $2 K \times 2 K$ nonsingular matrix. Next, define the following two selection matrices

$\mathbf{J}_{1}=\left[\mathbf{I}_{6 P-6}, \mathbf{0}_{(6 P-6) \times 6}\right], \mathbf{J}_{2}=\left[\mathbf{0}_{(6 P-6) \times 6}, \mathbf{I}_{6 P-6}\right]$

and let $\mathbf{B}_{1}=\mathbf{J}_{1} \mathbf{B}$ and $\mathbf{B}_{2}=\mathbf{J}_{2} \mathbf{B}$. The shift invariance structure in $\mathbf{B}$ indicates that

$$
\mathbf{B}_{2}=\mathbf{B}_{1} \Phi
$$

where

$$
\boldsymbol{\Phi}=\operatorname{diag}\left[e^{j \omega_{1,1} \Delta_{T}}, e^{j \omega_{1,2} \Delta_{T}}, \cdots, e^{j \omega_{K, 1} \Delta_{T}}, e^{j \omega_{K, 2} \Delta_{T}}\right]
$$

From (20) and (22), we obtain

$$
\mathbf{T}^{-1} \mathbf{\Phi} \mathbf{T}=\mathbf{E}_{1}^{\dagger} \mathbf{E}_{2}
$$

where $\mathbf{E}_{1}=\mathbf{J}_{1} \mathbf{E}_{s}$ and $\mathbf{E}_{2}=\mathbf{J}_{2} \mathbf{E}_{s}$. Consequentially, the ESPRIT's eigenvalues, i.e., the eigenvalues of $\mathbf{E}_{1}^{\dagger} \mathbf{E}_{2}$ equal the diagonal elements of $\boldsymbol{\Phi}$, and the ESPRIT's right eigenvectors constitute the columns of $\mathbf{T}$. Thus, the matrix $\mathbf{B}_{1}$ may be estimated as

$$
\hat{\mathbf{B}}_{1}=\frac{1}{2}\left\{\mathbf{E}_{1} \mathbf{T}^{-1}+\mathbf{E}_{2} \mathbf{T}^{-1} \boldsymbol{\Phi}^{-1}\right\}
$$


Note that the matrix $\mathbf{B}_{1}$ has the form

$$
\begin{aligned}
\mathbf{B}_{1} & =\left[\mathbf{Q}_{1}^{T}, \mathbf{Q}_{2}^{T}, \cdots, \mathbf{Q}_{P-1}^{T}\right]^{T} \\
& =\left[\mathbf{Q}^{T},(\mathbf{Q} \boldsymbol{\Phi})^{T}, \cdots,\left(\mathbf{Q} \boldsymbol{\Phi}^{(P-2)}\right)^{T}\right]^{T}
\end{aligned}
$$

where $\mathbf{Q}=\mathbf{M A}$. Therefore, the matrix $\mathbf{Q}$ can be estimated from $\hat{\mathbf{B}}_{1}$ as

$$
\hat{\mathbf{Q}}=\frac{1}{P-1} \sum_{p=1}^{P-1} \mathbf{Q}_{p} \Phi^{-(p-1)}
$$

It should be pointed out that the estimated $\mathbf{Q}$ would suffer the unknown scaling ambiguities of the columns. That is, the columns of the estimated $\hat{\mathbf{Q}}$ in fact satisfy

$$
\begin{aligned}
& \mathbf{q}_{2 k-1}=\alpha_{1, k} \mathbf{C v}_{1, k}, \quad \mathbf{q}_{2 k}=\alpha_{2, k} \mathbf{C v}_{2, k} \\
& \overline{\mathbf{q}}_{2 k-1}=\bar{\alpha}_{1, k} \mathbf{C v}_{2, k}, \quad \overline{\mathbf{q}}_{2 k}=\bar{\alpha}_{2, k} \mathbf{C v}_{1, k}
\end{aligned}
$$

where $\mathbf{q}_{2 k-1}, \mathbf{q}_{2 k}$ and $\overline{\mathbf{q}}_{2 k-1}, \overline{\mathbf{q}}_{2 k}, k=1, \cdots, K$, respectively, denote the top three and bottom three rows of the $(2 k-1)$ th and $(2 k)$ th columns of $\hat{\mathbf{Q}}, \alpha_{i, j}$ and $\bar{\alpha}_{i, j}$, $i=1,2, j=1, \cdots, K$ represent the unknown scalars. Note that since $q_{k} \neq 1, \alpha_{i, j}$ is in general unequal to $\bar{\alpha}_{i, j}$.

The scaling ambiguities can be easily eliminated in the proposed method. Using $\mathbf{q}_{2 k}=\alpha_{2, k} \mathbf{C v}_{2, k}$, we can form the following three equations:

$$
\begin{aligned}
& \alpha_{2, k}\left(-c_{1} \sin \phi_{k}+c_{2} \cos \phi_{k}\right)=q_{2 k, 1} \\
& \alpha_{2, k}\left(-c_{2} \sin \phi_{k}+c_{1} \cos \phi_{k}\right)=q_{2 k, 2} \\
& \alpha_{2, k}\left(-c_{2} \sin \phi_{k}+c_{2} \cos \phi_{k}\right)=q_{2 k, 3}
\end{aligned}
$$

where $q_{2 k, 1}, q_{2 k, 2}$, and $q_{2 k, 3}$ are, respectively, the first, second, and third entries of $\mathbf{q}_{2 k}$. Solving these three equations yields the azimuth angle and coupling coefficient estimates:

$$
\begin{gathered}
\hat{\phi}_{k}=\arctan \left(\frac{q_{2 k, 1}-q_{2 k, 3}}{q_{2 k, 3}-q_{2 k, 2}}\right) \\
\hat{c}=c_{2} / c_{1}=\frac{1}{2}\left(\frac{q_{2 k, 1} \cos \hat{\phi}_{k}+q_{2 k, 2} \sin \hat{\phi}_{k}}{q_{2 k, 1} \sin \hat{\phi}_{k}+q_{2 k, 2} \cos \hat{\phi}_{k}}\right. \\
\left.+\frac{q_{2 k, 3} \cos \hat{\phi}_{k}}{q_{2 k, 2} \cos \hat{\phi}_{k}+q_{2 k, 3} \sin \hat{\phi}_{k}-q_{2 k, 2} \sin \hat{\phi}_{k}}\right)
\end{gathered}
$$

With the estimation of the coupling coefficient $\hat{c}$, we can construct an estimate of the mutual coupling matrix $\hat{\mathbf{C}}$ as

$$
\hat{\mathbf{C}}=\left[\begin{array}{lll}
1 & \hat{c} & \hat{c} \\
\hat{c} & 1 & \hat{c} \\
\hat{c} & \hat{c} & 1
\end{array}\right]
$$

It is easy to see that the matrix product $\hat{\mathbf{C}} \mathbf{C}$ becomes a scaled identity matrix. This means that the mutual coupling coefficients, which constitute the non-diagonal elements of $\mathbf{C}$, are completely eliminated. With the estimation of $\hat{\phi}_{k}$ and $\hat{c}$, using $\overline{\mathbf{q}}_{2 k}=\bar{\alpha}_{2, k} \mathbf{C v}_{1, k}$, we can form the following three equations:

$\bar{\alpha}_{2, k}\left(\cos \hat{\phi}_{k} \cos \theta+\hat{c} \sin \hat{\phi}_{k} \cos \theta-\hat{c} \sin \theta\right)=\bar{q}_{2 k, 1}$

$\bar{\alpha}_{2, k}\left(\hat{c} \cos \hat{\phi}_{k} \cos \theta+\sin \hat{\phi}_{k} \cos \theta-\hat{c} \sin \theta\right)=\bar{q}_{2 k, 2}$

$\bar{\alpha}_{2, k}\left(\hat{c} \cos \hat{\phi}_{k} \cos \theta+\hat{c} \sin \hat{\phi}_{k} \cos \theta-\sin \theta\right)=\bar{q}_{2 k, 3}$

Solving these three equations leads to the elevation angle estimates

$$
\begin{aligned}
\hat{\theta}_{k}= & \arctan \left(\hat{c}\left(\cos \hat{\phi}_{k}+\sin \hat{\phi}_{k}\right)-\frac{\bar{q}_{2 k, 3}}{\bar{q}_{2 k, 1}-\bar{q}_{2 k, 2}}\right. \\
& \left.\times(1-\hat{c})\left(\cos \hat{\phi}_{k}-\sin \hat{\phi}_{k}\right)\right)
\end{aligned}
$$

Note that the estimation of $\hat{\theta}_{k}$ and $\hat{\phi}_{k}$ are automatically paired without any additional processing.

In practice, apart from the scaling ambiguities, the estimated $\hat{\mathbf{Q}}$ may also suffer from some permutation ambiguities. In this case, $\mathbf{q}_{2 k}$ may not be the estimate of $\alpha_{2, k} \mathbf{C v}_{2, k}$. Thus, the estimation of $\hat{\phi}_{k}$ and $\hat{c}$ obtained by using (33) and (34) from $\mathbf{q}_{2 k}$ may be erroneous. These may further result in the erroneous estimation of $\hat{\theta}_{k}$. Unlike the scaling ambiguities, the permutation ambiguities are not resolvable. Here, we provide a solution to deal with this permutation ambiguity problem as follows: first, for all $k=1, \cdots, 2 K$, obtain a set of $2 K$ different azimuth angle estimates from $\mathbf{q}_{k}$. Each of these $2 K$ azimuth angle estimates is then used to produce its own coupling coefficient and elevation angle estimates. Thus, the $k$ th azimuth angle, elevation angle, and coupling coefficient estimates are automatically matched. We know that only a set of $K$ estimates are true estimates. Theoretically, the $K$ true coupling coefficient estimates are identical, while the $K$ erroneous coupling coefficient estimates are, in general, distinct from one another and from the $K$ true estimates. Therefore, we can take homogeneity in coupling coefficient estimates as a criterion for determining the true estimates of the angles and coupling coefficients, i.e., we take a set of $K$ angle estimates associated with $K$ identical coupling coefficient estimates as the true estimates. Without loss of generality, let us assume that the first $K$ estimates are true and the last $K$ estimates are erroneous; then, we have $\hat{c}_{1}=\cdots=\hat{c}_{K}=\hat{c} \neq \hat{c}_{K+1} \neq \cdots \neq \hat{c}_{2 K}$. Finally, we obtain the estimates $\left(\hat{\theta}_{k}, \hat{\phi}_{k}\right), k=1, \cdots, K$ as the angle estimates of the $K$ signals. 


\subsection{Remarks}

In the presence of noise, the estimation procedures in Section 3.2 becomes approximate. Specially, with noise, the set of $K$ coupling coefficient estimates are in general different. Nevertheless, we can search for a set of $K$ coupling coefficient estimates with "most similar values" as the "identical" estimates.

Also note that the vector cross product estimator has been widely used for direction finding with a singlevector sensor [2, 3, 7]. However, this estimator cannot be exploited directly in the presence of mutual coupling among the vector sensor components. Obviously, with the estimation of $\hat{c}$, the vector sensor can be calibrated by using the calibration matrix defined as $\hat{\mathbf{M}}=\mathbf{I}_{2} \otimes$ $\hat{\mathbf{C}}$. Therefore, the vector cross product estimator can be applied to the calibrated data matrix $\hat{\mathbf{M Z}}$ to extract the angle estimates of the incoming signals. Although the proposed method is designed for vector sensors with mutual coupling, it can also be applied to ideal vector sensors, where the measurement of each component is independent of the others.

The proposed method shares all the advantages indicated in [3]. For example, it offers automatically paired azimuth and elevation angle estimates, does not restrict $\Delta_{T}$ to be constricted by the Nyquist sampling rate, does not need the signal frequencies to be known a priori, and suffers no frequency-DOA ambiguity. It should be noted that the method in [3] assumes CP signals, whereas the proposed method assumes IP ones.

Lastly, it should be pointed out that the application of ESPRIT technique for vector sensor mutual coupling calibration has been studied in works [22] and [23]. However, the differences between these two works and the present work are that (1) the former requires a coupling-free auxiliary vector sensor and design of a reference signal, while the latter does not, (2) the former does not apply the temporally smoothing technique to improve the identifiability limit of a vector sensor, and (3) the former assumes the incoming signals are completely polarized, while the latter considers the incompletely polarized signals.

\section{Cramér-Rao bound}

This section derives the CRB for the problem considered above, under the assumptions made in Section 2. Further, the following assumptions are added: (i) we assume $c_{1}=1$ and $c_{2}=c \neq 1$ due to the fact that the vector sensor can be calibrated with the estimation of $c=c_{2} / c_{1}$. (ii) Since the spatial phase factor $q_{k}$ between the dipole triad and loop triad is considered as a scalar constant in the proposed method, we assume $q_{k}=1, k=1, \cdots, K$ for convenience. (iii) The energies, frequencies and initial phases of the signals are presumed as known constants. (iv) The $6 \times 6$ noise covariance matrix $\mathbf{R}_{n}$ is presumed as unknown, deterministic, and diagonal with all diagonal elements equal to $\sigma^{2}$. Hence, the observation data satisfies the following model:

$$
\mathbf{z}\left(n \Delta_{T}\right) \sim \mathcal{C N}\left(\boldsymbol{\mu}(n), \sigma^{2} \mathbf{I}_{6}\right)
$$

where $\boldsymbol{\mu}(n)=\sum_{k=1}^{K} \mathbf{M A}_{k} \mathbf{s}_{k}\left(n \Delta_{T}\right), n=1, \cdots, N$.

Define a $(2 K+2) \times 1$ unknown parameter vector $\boldsymbol{\theta}=$ $\left[\mathbf{c}^{T}, \boldsymbol{\theta}_{1}^{T}, \cdots, \boldsymbol{\theta}_{K}^{T}\right]^{T}$, where $\mathbf{c}=[\operatorname{Re}(c), \operatorname{Im}(c)]^{T}$ and $\boldsymbol{\theta}_{k}=$ $\left[\theta_{k}, \phi_{k}\right]^{T}$. Then, the $(2 K+2) \times(2 K+2)$ Fisher information matrix (FIM) is given by

$$
\mathbf{J}(\boldsymbol{\theta})=2 \sum_{n=1}^{N}\left\{\operatorname{Re}\left[\left(\frac{\partial \boldsymbol{\mu}(n)}{\partial \boldsymbol{\theta}}\right)^{H} \mathbf{R}_{n}^{-1}\left(\frac{\partial \boldsymbol{\mu}(n)}{\partial \boldsymbol{\theta}}\right)\right]\right\}
$$

where the elements of $\partial \boldsymbol{\mu}(n) / \partial \boldsymbol{\theta}$ are

$$
\begin{gathered}
\frac{\partial \boldsymbol{\mu}(n)}{\partial \operatorname{Re}(c)}=\sum_{k=1}^{K}\left(\mathbf{I}_{2} \otimes \mathbf{C}^{\prime}\right) \mathbf{A}_{k} \mathbf{s}_{k}\left(n \Delta_{T}\right) \\
\frac{\partial \boldsymbol{\mu}(n)}{\partial \operatorname{Im}(c)}=j \sum_{k=1}^{K}\left(\mathbf{I}_{2} \otimes \mathbf{C}^{\prime}\right) \mathbf{A}_{k} \mathbf{s}_{k}\left(n \Delta_{T}\right) \\
\frac{\partial \boldsymbol{\mu}(n)}{\partial \theta_{k}}=\left(\mathbf{I}_{2} \otimes \mathbf{C}\right) \mathbf{A}_{\theta_{k}}^{\prime} \mathbf{s}_{k}\left(n \Delta_{T}\right) \\
\frac{\partial \boldsymbol{\mu}(n)}{\partial \phi_{k}}=\left(\mathbf{I}_{2} \otimes \mathbf{C}\right) \mathbf{A}_{\phi_{k}}^{\prime} \mathbf{s}_{k}\left(n \Delta_{T}\right)
\end{gathered}
$$

where

$$
\begin{gathered}
\mathbf{C}^{\prime}=\left[\begin{array}{lll}
0 & 1 & 1 \\
1 & 0 & 1 \\
1 & 1 & 0
\end{array}\right] \\
\mathbf{A}_{\theta_{k}}^{\prime}=\left[\begin{array}{cc}
\mathbf{v}_{1, k}^{\theta} & \mathbf{0} \\
\mathbf{0} & -\mathbf{v}_{1, k}^{\theta}
\end{array}\right] \\
\mathbf{A}_{\phi_{k}}^{\prime}=\left[\begin{array}{cc}
\mathbf{v}_{1, k}^{\phi} & \mathbf{v}_{2, k}^{\phi} \\
\mathbf{v}_{2, k}^{\phi} & -\mathbf{v}_{1, k}^{\theta}
\end{array}\right]
\end{gathered}
$$

with $\mathbf{v}_{1, k}^{\theta}=\left[-\cos \phi_{k} \sin \theta_{k},-\sin \phi_{k} \sin \theta_{k},-\cos \theta_{k}\right]^{T}$, $\mathbf{v}_{1, k}^{\phi}=\left[-\sin \phi_{k} \cos \theta_{k}, \cos \phi_{k} \cos \theta_{k}, 0\right]^{T}$, and $\mathbf{v}_{2, k}^{\phi}=$ $\left[-\cos \phi_{k},-\sin \phi_{k}, 0\right]^{T}$. Note that $\mathbf{J}(\boldsymbol{\theta})$ can be expressed in a block matrix form as

$$
\mathbf{J}(\boldsymbol{\theta})=\left[\begin{array}{cccc}
\mathbf{J}_{\mathbf{c}, \mathbf{c}} & \mathbf{J}_{\mathbf{c}, \boldsymbol{\theta}_{1}} & \cdots & \mathbf{J}_{\mathbf{c}, \boldsymbol{\theta}_{K}} \\
\mathbf{J}_{\boldsymbol{\theta}_{1}, \mathbf{c}} & \mathbf{J}_{\boldsymbol{\theta}_{1}, \boldsymbol{\theta}_{1}} & \cdots & \mathbf{J}_{\boldsymbol{\theta}_{1}, \boldsymbol{\theta}_{K}} \\
\vdots & \vdots & \cdots & \vdots \\
\mathbf{J}_{\boldsymbol{\theta}_{K}, \mathbf{c}} & \mathbf{J}_{\boldsymbol{\theta}_{K}, \boldsymbol{\theta}_{1}} & \cdots & \mathbf{J}_{\boldsymbol{\theta}_{K}, \boldsymbol{\theta}_{K}}
\end{array}\right]
$$

where all the blocks are of size $2 \times 2$. Using the fact that for large $N, \frac{1}{N} \sum_{n=1}^{N} s_{i}\left(n \Delta_{T}\right) s_{j}^{*}\left(n \Delta_{T}\right)=\delta_{i j}$, where $\delta_{i j}$ denotes the Dirac delta, $\mathbf{J}(\boldsymbol{\theta})$ would asymptotically have the form

$$
\mathbf{J}(\boldsymbol{\theta})=\left[\begin{array}{cc}
\mathbf{J}_{\mathbf{c}, \mathbf{c}} & \mathbf{J}_{\mathbf{c}, \boldsymbol{\Theta}} \\
\mathbf{J}_{\Theta, \mathbf{c}} & \mathbf{J}_{\Theta, \Theta}
\end{array}\right]
$$


where

$$
\begin{gathered}
\mathbf{J}_{\boldsymbol{\Theta}, \mathbf{c}}=\mathbf{J}_{\mathbf{c}, \boldsymbol{\Theta}}^{T}=\left[\mathbf{J}_{\mathbf{c}, \boldsymbol{\theta}_{1}}, \cdots, \mathbf{J}_{\mathbf{c}, \boldsymbol{\theta}_{K}}\right] \\
\mathbf{J}_{\boldsymbol{\Theta}, \boldsymbol{\Theta}}=\left[\begin{array}{lll}
\mathbf{J}_{\boldsymbol{\theta}_{1}, \boldsymbol{\theta}_{1}} & & \\
& \ddots & \\
& & \mathbf{J}_{\boldsymbol{\theta}_{K}, \boldsymbol{\theta}_{K}}
\end{array}\right]
\end{gathered}
$$

Finally, computing the inverse of the FIM, we obtain the following closed-form expression for the CRB:

$$
\begin{gathered}
\mathrm{CRB}(\mathbf{c})=\frac{\sigma^{2}}{2 N}\left(\mathbf{J}_{\mathbf{c}, \mathbf{c}}-\sum_{k=1}^{K}\left(\mathbf{J}_{\mathbf{c}, \boldsymbol{\theta}_{k}} \mathbf{J}_{\boldsymbol{\theta}_{k}, \boldsymbol{\theta}_{k}}^{-1} \mathbf{J}_{\boldsymbol{\theta}_{k}, \mathbf{c}}\right)\right)^{-1} \\
\mathrm{CRB}(\boldsymbol{\Theta})=\frac{\sigma^{2}}{2 N}\left(\mathbf{J}_{\boldsymbol{\Theta}, \boldsymbol{\Theta}}-\mathbf{J}_{\boldsymbol{\Theta}, \mathbf{c}} \mathbf{J}_{\mathbf{c}, \mathbf{c}}^{-1} \mathbf{J}_{\mathbf{c}, \boldsymbol{\Theta}}\right)^{-1}
\end{gathered}
$$

where

$$
\begin{gathered}
\mathbf{J}_{\mathbf{c}, \mathbf{c}}=J_{\operatorname{Im}(c), \operatorname{Im}(c)} \mathbf{I}_{2}=J_{\operatorname{Re}(c), \operatorname{Re}(c)} \mathbf{I}_{2} \\
\mathbf{J}_{\boldsymbol{\theta}_{k}, \mathbf{c}}=\mathbf{J}_{\mathbf{c}, \boldsymbol{\theta}_{k}}=\left[\begin{array}{ll}
J_{\operatorname{Re}(c), \theta_{k}} & J_{\operatorname{Re}(c), \phi_{k}} \\
J_{\operatorname{Im}(c), \theta_{k}} & J_{\operatorname{Im}(c), \phi_{k}}
\end{array}\right] \\
\mathbf{J}_{\boldsymbol{\theta}_{k}, \boldsymbol{\theta}_{k}}=\left[\begin{array}{ll}
J_{\theta_{k}, \theta_{k}} & J_{\theta_{k}, \phi_{k}} \\
J_{\phi_{k}, \theta_{k}} & J_{\phi_{k}, \phi_{k}}
\end{array}\right]
\end{gathered}
$$

with

$$
J_{\operatorname{Re}(c), \operatorname{Re}(c)}=2 \sum_{k=1}^{K}\left(\left(\mathbf{C}^{\prime} \mathbf{v}_{1, k}\right)^{H}\left(\mathbf{C}^{\prime} \mathbf{v}_{1, k}\right)+\left(\mathbf{C}^{\prime} \mathbf{v}_{2, k}\right)^{H}\left(\mathbf{C}^{\prime} \mathbf{v}_{2, k}\right)\right)
$$

$$
\begin{aligned}
& J_{\operatorname{Re}(c), \theta_{k}}=2\left(\left(\mathbf{C}^{\prime} \mathbf{v}_{1, k}\right)^{H}\left(\operatorname{Re}(\mathbf{C}) \mathbf{v}_{1, k}^{\theta}\right)\right) \\
& J_{\operatorname{Re}(c), \phi_{k}}=2\left(\left(\mathbf{C}^{\prime} \mathbf{v}_{1, k}\right)^{H}\left(\operatorname{Re}(\mathbf{C}) \mathbf{v}_{1, k}^{\phi}\right)+\left(\mathbf{C}^{\prime} \mathbf{v}_{2, k}\right)^{H}\left(\operatorname{Re}(\mathbf{C}) \mathbf{v}_{2, k}^{\phi}\right)\right)
\end{aligned}
$$

$$
J_{\operatorname{Im}(c), \theta_{k}}=2\left(\left(\mathbf{C}^{\prime} \mathbf{v}_{1, k}\right)^{H}\left(\operatorname{Im}(\mathbf{C}) \mathbf{v}_{1, k}^{\theta}\right)\right)
$$

$$
J_{\operatorname{Im}(c), \phi_{k}}=2\left(\left(\mathbf{C}^{\prime} \mathbf{v}_{1, k}\right)^{H}\left(\operatorname{Im}(\mathbf{C}) \mathbf{v}_{1, k}^{\phi}\right)+\left(\mathbf{C}^{\prime} \mathbf{v}_{2, k}\right)^{H}\left(\operatorname{Im}(\mathbf{C}) \mathbf{v}_{2, k}^{\phi}\right)\right)
$$

$$
\begin{gathered}
J_{\theta_{k}, \theta_{k}}=2\left(\left(\mathbf{C} \mathbf{v}_{1, k}^{\theta}\right)^{H}\left(\mathbf{C} \mathbf{v}_{1, k}^{\theta}\right)\right) \\
J_{\theta_{k}, \phi_{k}}=J_{\phi_{k}, \theta_{k}}=2\left(\left(\mathbf{C} \mathbf{v}_{1, k}^{\theta}\right)^{H}\left(\mathbf{C} \mathbf{v}_{1, k}^{\phi}\right)\right) \\
J_{\phi_{k}, \phi_{k}}=2\left(\left(\mathbf{C} \mathbf{v}_{1, k}^{\phi}\right)^{H}\left(\mathbf{C v}_{1, k}^{\phi}\right)+\left(\mathbf{C v}_{2, k}^{\phi}\right)^{H}\left(\mathbf{C v}_{2, k}^{\phi}\right)\right)(65)
\end{gathered}
$$

\section{Simulation results and discussion}

\subsection{Simulation results}

In this section, we provide simulation results to illustrate the performance of the proposed ESPRIT-based method. In all the simulations, the vector sensor is assumed to be spatially collocated with the mutual coupling model defined in (12). The mutual coupling coefficient used is $c=0.1 e^{-j \pi / 4}$. The additive noise is assumed to be spatial white complex Gaussian, and the SNR is defined relative to each signal. The result in each of the examples below is obtained from 500 independent Monte-Carlo trials. For comparison purposes, three different methods are considered. The first method is to apply the vector cross product estimator to the measured data directly. This method is hereafter referred to as "VCP Estimator without calibration." The second method is based on the condition that the mutual coupling coefficient is known a priori, and the vector cross product estimator is applied to the perfectly calibrated data. This method is referred to as "VCP Estimator perfect calibration." The third method is the auxiliary sensor calibration method presented in [22]. The performance metric used is the root mean squared error (RMSE) of the two signals.

In the first example, we present the scatter diagram of the azimuth-elevation angle estimates of four source signals in Fig. 2 to show the effectiveness of the temporal smoothing technique. The angles of signals are set as $\left(\theta_{1}, \cdots, \theta_{4}\right)=\left(24.35^{\circ}, 12.92^{\circ}, 21.13^{\circ}, 39.82^{\circ}\right)$ and $\left(\phi_{1}, \cdots, \phi_{4}\right)=\left(75.96^{\circ}, 26.57^{\circ}, 33.69^{\circ}, 51.34^{\circ}\right)$. The SNR is assumed to be $25 \mathrm{~dB}$ and $N=15$ data samples are used. $P=8$ is used for performing the temporal smoothing processing. From the figure, we can see that the proposed

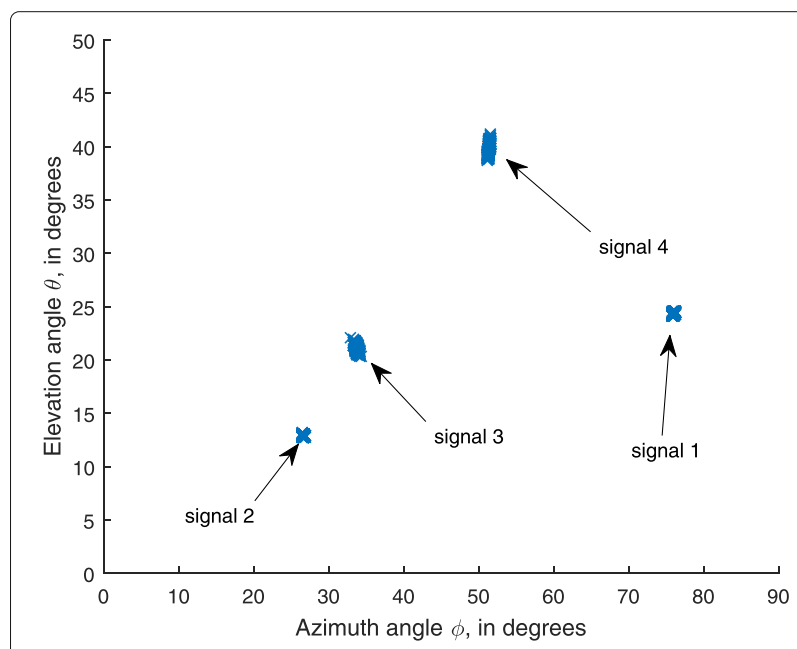

Fig. 2 Angle estimation result for four source signals. The angles of signals are set as: $\left(\theta_{1}, \cdots, \theta_{4}\right)=\left(24.35^{\circ}, 12.92^{\circ}, 21.13^{\circ}, 39.82^{\circ}\right)$ and $\left(\phi_{1}, \cdots, \phi_{4}\right)=\left(75.96^{\circ}, 26.57^{\circ}, 33.69^{\circ}, 51.34^{\circ}\right)$. Five hundred independent experiments are conducted 
method successfully resolves the four signals, as stated in Theorem 1: $N \geq 4 K-1$.

In the second example, we study the performance of the proposed method with different temporal smoothing dimension $P$. Two equal-power narrowband uncorrelated monochromatic IP signals with randomly chosen digital frequencies impinge upon the vector sensor with angles: $\theta_{1}=21.13^{\circ}, \phi_{1}=33.69^{\circ}$ and $\theta_{2}=39.82^{\circ}, \phi_{2}=51.34^{\circ}$. The number of snapshots and SNR used are, respectively, $N=200$ and SNR $=25 \mathrm{~dB}$. The RMSEs of angle estimates as a function of the value $P$, varying from 8 to 40 , are shown in Fig. 3. We see from the figure that the estimation errors remain almost unchanged with the increasing of value $P$.

In the third example, we compare the performance of the proposed method with the VCP Estimator without calibration, VCP Estimator perfect calibration, and auxiliary sensor calibration method. The simulation conditions are similar to those in the second example, except that the SNR is varying from 0 to $40 \mathrm{~dB}$ in steps of $5 \mathrm{~dB}$. The RMSEs of angle estimates are shown in Fig. 4, where the CRBs are also plotted for comparison. We see from the figure that the proposed method has a performance significantly superior to that of the VCP Estimator without calibration. For azimuth angle estimation, the proposed method outperforms the VCP Estimator perfect calibration and auxiliary sensor calibration method at all SNRs. For elevation angle estimation, the RMSEs of the proposed method are slightly greater than those of the VCP Estimator perfect calibration and auxiliary sensor calibration method. This phenomenon can be explained as follows. Referring back to (33), (34), and (39), the proposed method obtain the estimates of azimuth angles, coupling coefficients and elevation angles in a successive way, and the estimation of the elevation angles is based on the previous estimations of the azimuth angles and the mutual coefficient. Consequently, the errors in the azimuth angle and coupling coefficient estimates will produce additional errors in the elevation angle estimates. Note that, with the estimation of coupling coefficient, the estimation performance for elevation angle can be further improved by statistically optimal algorithms such as maximum likelihood algorithm and subspace fitting algorithm. Incidentally, since the estimate errors are a bit bigger than $\mathrm{CRB}$, the proposed method might be biased.

In the fourth example, we assess the performance of the proposed method versus the number of snapshots. The simulation conditions are similar to those in the second example, except that the SNR is set at $20 \mathrm{~dB}$ and the number of snapshots is varied from $N=20$ to $N=$ 2000. The RMSEs of the angle estimates are plotted in Fig. 5, and compared with those of the VCP Estimator without calibration, VCP Estimator perfect calibration, auxiliary sensor calibration method, and the CRBs. We see from Fig. 5 that the results are similar to those of the first example. The RMSEs of the proposed method decrease monotonically with the number of snapshots. Moveover, for azimuth angle estimation, the RMSEs of the proposed method are lower than those of the VCP Estimator perfect calibration and auxiliary sensor calibration method.

In the last example, we investigate the RMSEs of angle estimates for the proposed method against the coupling level, which is represented by coupling coefficient $c=$ $\rho e^{j \pi / 4}$, varying low $(\rho=0.1)$ to high $(\rho=0.9)$. One IP signal with $\theta=21.13^{\circ}, \phi=33.69^{\circ}$, and randomly chosen digital frequencies impinges upon the sensor. The number of snapshots and SNR used are, respectively, $N=200$ and SNR $=25 \mathrm{~dB}$. The RMSEs of angle estimates as a function of the value $\rho$ are shown in Fig. 6. We see from the figure that the proposed method can handle both low and high coupling levels, since the estimation errors remain almost constant with the increasing of coupling coefficient $\rho$.
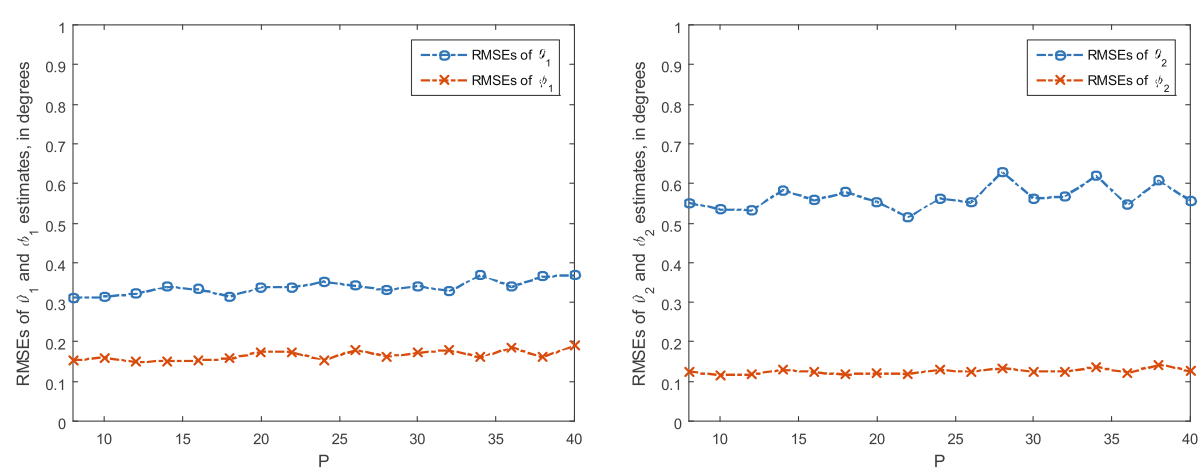

Fig. 3 RMSEs of angle estimates against $P$. Two uncorrelated signals with $\theta_{1}=21.13^{\circ}, \phi_{1}=33.69^{\circ}$ and $\theta_{2}=39.82^{\circ}, \phi_{2}=51.34^{\circ}$ impinge upon a single-vector sensor. Five hundred independent experiments are conducted 

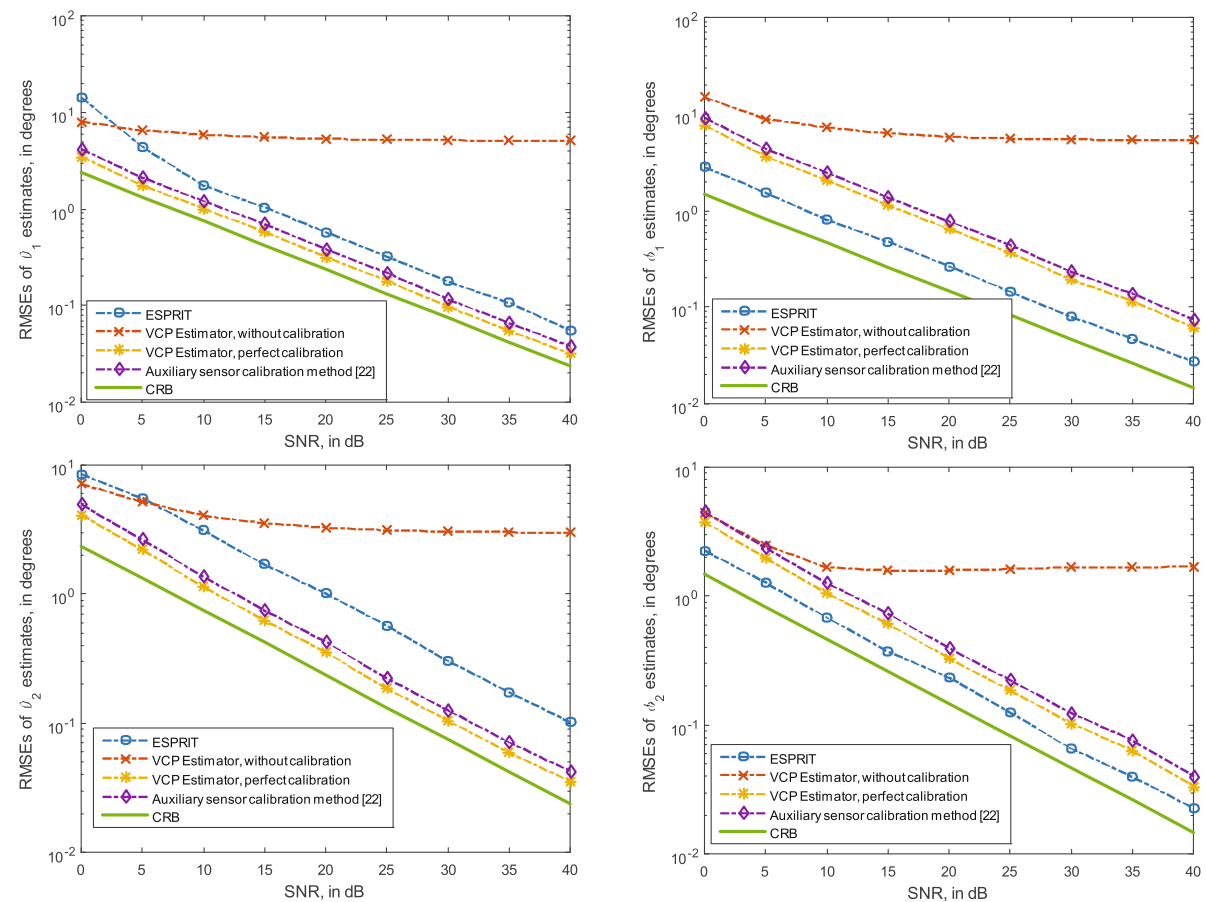

Fig. 4 RMSEs of angle estimates against SNR. Two uncorrelated signals with $\theta_{1}=21.13^{\circ}, \phi_{1}=33.69^{\circ}$ and $\theta_{2}=39.82^{\circ}, \phi_{2}=51.34^{\circ} \mathrm{impinge} \mathrm{upon} \mathrm{a}$ single-vector sensor. Five hundred independent experiments are conducted
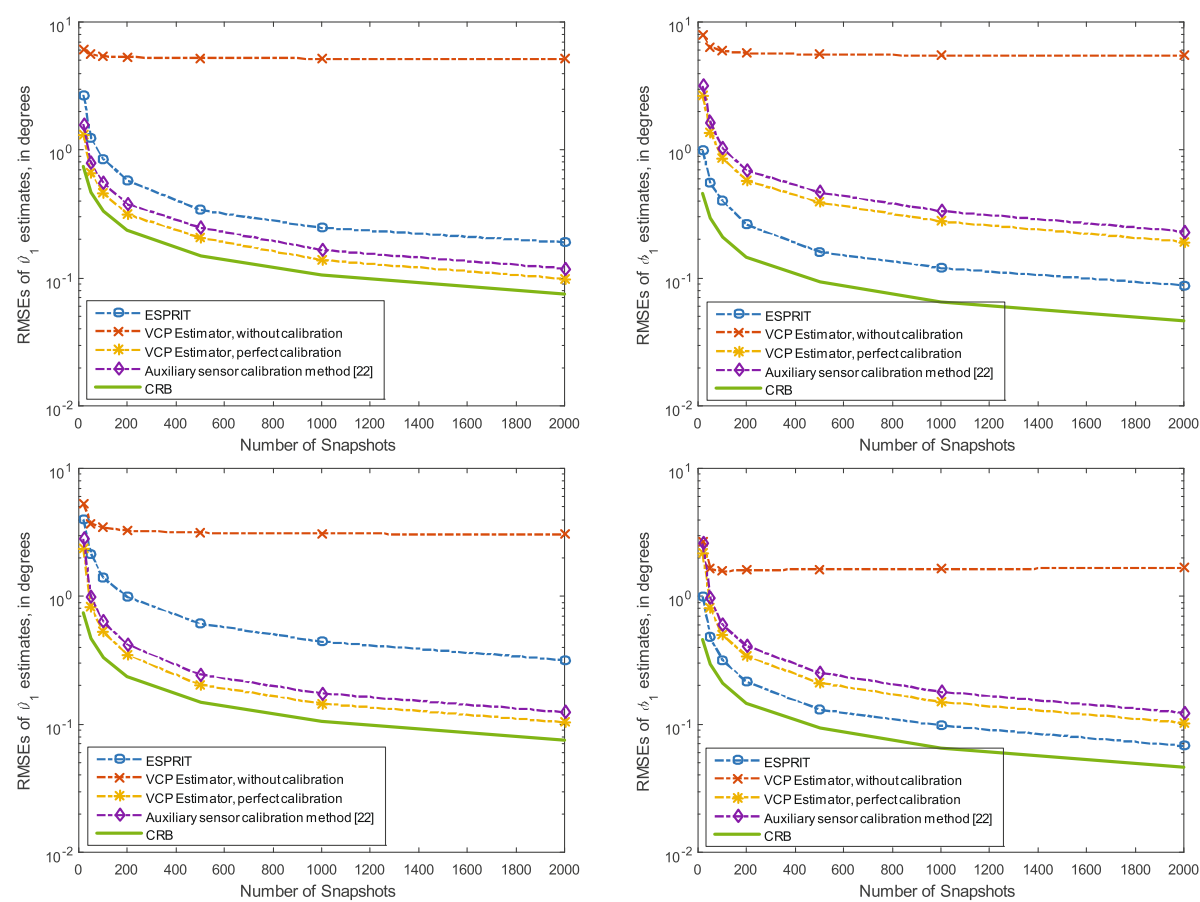

Fig. 5 RMSEs of angle estimates against snapshots. Two uncorrelated signals with $\theta_{1}=21.13^{\circ}, \phi_{1}=33.69^{\circ}$ and $\theta_{2}=39.82^{\circ}, \phi_{2}=51.34^{\circ}$ impinge upon a single-vector sensor. Five hundred independent experiments are conducted 


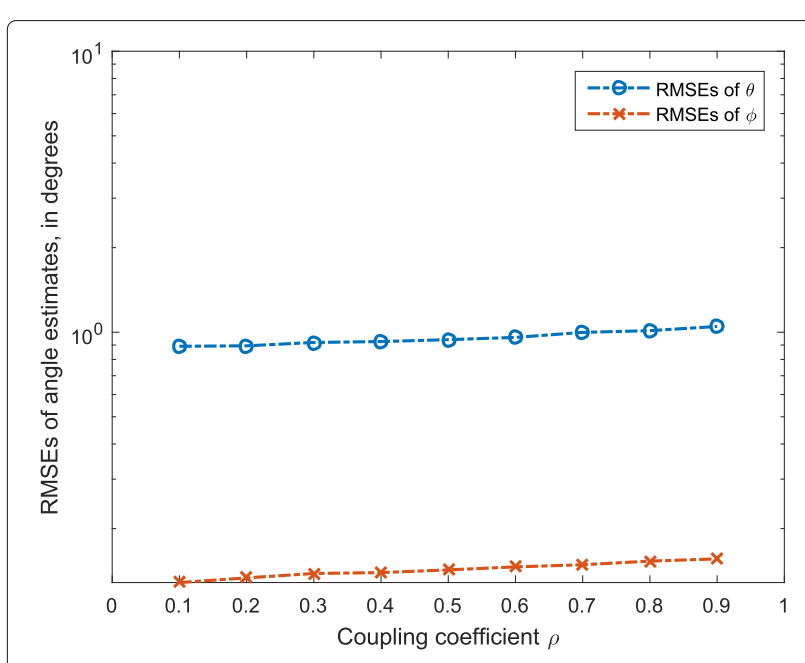

Fig. 6 RMSEs of $\theta$ and $\phi$ estimates versus coupling coefficient $\rho$. Five hundred independent experiments are conducted

\subsection{Discussion}

The proposed method is valid only for the case of $K \geq 2$. If there is only one incoming signal, this method cannot be used. In this case, the temporal smoothing process is not necessary, and a method for determining the true estimates is formulated as follows: let $\mathbf{E}_{n}$ be the $6 \times 4$ noise-subspace eigenvector matrix whose four columns are the $6 \times 1$ noise-subspace eigenvectors associated with four smallest eigenvalues of $\mathbf{Z} \mathbf{Z}^{H}$. For the two sets of estimates $\left\{\left(\hat{c}_{1}, \hat{\theta}_{1}, \hat{\phi}_{1}\right),\left(\hat{c}_{2}, \hat{\theta}_{2}, \hat{\phi}_{2}\right)\right\}$, we may determine as to which one is the true one by first constructing the matrices $\mathbf{I}_{2} \otimes \hat{\mathbf{C}}_{1} \hat{\mathbf{A}}_{1}$ and $\mathbf{I}_{2} \otimes \hat{\mathbf{C}}_{2} \hat{\mathbf{A}}_{2}$, and then taking $\left(\hat{c}_{1}, \hat{\theta}_{1}, \hat{\phi}_{1}\right)$ to be the true estimates if

$$
\left\|\left[\mathbf{I}_{2} \otimes \hat{\mathbf{C}}_{1} \hat{\mathbf{A}}_{1}\right]^{H} \mathbf{E}_{n}\right\|<\left\|\left[\mathbf{I}_{2} \otimes \hat{\mathbf{C}}_{2} \hat{\mathbf{A}}_{2}\right]^{H} \mathbf{E}_{n}\right\|
$$

or $\left(\hat{c}_{2}, \hat{\theta}_{2}, \hat{\phi}_{2}\right)$ otherwise, where $\|\cdot\|$ denotes the Frobenius norm.

\section{Conclusions}

The present paper has considered, for the first time, the direction finding using a single-vector sensor in the presence of mutual coupling. The temporal smoothing technique has been applied to improve the identifiability limit of a single vector. In particular, sufficient conditions for constructing temporally smoothed matrices to resolve $K>2$ incompletely polarized (IP) monochromatic signals with a single vector sensor have been established. An efficient ESPRIT-based method, which does not require any calibration sources or iterative operations, has been developed to jointly estimate the azimuth-elevation angles and the mutual coupling coefficients. The CRB for the considered problem has also been derived. Simulation results have been presented showing the superiorities of the proposed method.

\section{Acknowledgements}

The authors would like to thank the anonymous reviewers for their valuable comments and suggestions that helped improve the quality of this manuscript.

\section{Funding}

This work was supported by the National Natural Science Foundation of China (no. 61771302) and Shanghai Association of Science and Technology (no. 16511103004)

\section{Availability of data and materials}

The datasets generated and/or analyzed during the current study are not publicly available but are available from the corresponding author on reasonable request.

\section{Authors' contributions}

All authors contributed extensively to the study presented in this manuscript. TS presented the main idea, carried out the simulation, interpreted the results, and wrote the paper. KW and JH conceived of the experiments. They also provided many valuable suggestions to this study. ZL supervised the main idea and edited the manuscript. All authors read and approved the final manuscript.

\section{Authors' information}

Ting Shu received the B.Sc. and M.Sc. degrees from Nanjing University of Science and Technology, Nanjing, China, in 2004 and 2006, respectively, both in electrical engineering. He received the Ph.d degrees in electrical engineering from Shanghai Jiao Tong University, Shanghai, China, in 2010. From 2010 to 2011, he worked as the system engineer in the Wireless Department of Huawei Technologies, Co., Ltd., responsible for the HSDPA baseband system design for the UMTS evolution. He joins SJTU in July, 2011. He is currently an assistant professor in electrical engineering at Shanghai Jiao Tong University. His current research interests include the hardware-in-loop simulation of radar and EW systems, R\&D of radar realtime signal processing, and adaptive array for phased array radar system. (Email: tingshu@sjtu.edu.cn) Kun Wang received the B.S. and Ph.D. degrees from Nanjing University of Science and Technology (NJUST), Nanjing, Jiangsu, China, in 2008 and 2017, respectively, both in electrical engineering. He is now a postdoctoral fellow with the Department of Electrical Engineering, Shanghai Jiaotong University, Shanghai, China. His research interests include sensor-array signal processing, statistical signal processing and their applications to sensors/radar networking. Jin He received his B.S. degree in information engineering from Tongji University, Shanghai, China in 2001 and his M.S. and Ph.D. degrees in electrical engineering from Nanjing University of Science and Technology (NJUST), Nanjing, Jiangsu, China in 2004 and 2007, respectively. From 2007 to 2009 he was a postdoctoral fellow with the Department of Electrical Engineering, NJUST, where he was the program director and was responsible for the China Postdoctoral Science Projects. From 2009 to 2011 he was a postdoctoral fellow with the Department of Electrical and Computer Engineering, Concordia University, Montreal, Quebec, Canada. From 2012 to 2015 he was a research engineer with the Shanghai Aerospace Electronic Technology Institute, Shanghai, China. He is now with the Shanghai Key Laboratory of Intelligent Sensing and Recognition, Department of Electronic Engineering, Shanghai Jiaotong University, Shanghai, China. Also he has served as a peer-reviewer for various IEEE/IET research journals since 2007. His research interests include array signal processing, statistical signal processing, non-Gaussian signal processing, and their applications.

Zhong Liu received the B.S. degree from Anhui University, Hefei, Anhui, China, in 1983 and the M.S. and Ph.D. degrees from University of Electronic Science and Technology of China, Chengdu, Sichuan, China, in 1985 and 1988, all in electrical engineering. He was a Postdoctoral Fellow at Kyoto University, Kyoto, Japan, from 1991 to 1993 and a visiting scholar at the Chinese University of HongKong, Hong Kong, from 1997 to 1998. Currently, he is a Professor in the Department of Electronic Engineering, Nanjing University of Science and Technology. His research interests include chaos and information dynamics, signal processing, radar and communication techniques.

\section{Competing interests}

The authors declare that they have no competing interests.

\section{Publisher's Note}

Springer Nature remains neutral with regard to jurisdictional claims in published maps and institutional affiliations. 


\begin{abstract}
Author details
${ }^{1}$ Shanghai Key Laboratory of Intelligent Sensing and Recognition, Department of Electronic Engineering, Shanghai Jiaotong University, Shanghai 200240, People's Republic of China. ${ }^{2}$ Department of Electronic Engineering, Nanjing University of Science and Technology, Nanjing 210094, People's Republic of China.
\end{abstract}

Received: 28 July 2017 Accepted: 12 February 2018

Published online: 27 February 2018

\section{References}

1. J Li, Direction and polarization estimation using arrays with small loops and short dipoles. IEEE Trans. Antennas Propag. 41, 379-387 (1993)

2. A Nehorai, E Paldi, Vector-sensor array processing for electromagnetic source localization. IEEE Trans. Signal Process. 42, 376-398 (1994)

3. KT Wong, MD Zoltowski, Uni-vector-sensor ESPRIT for multisource azimuth, elevation, and polarization estimation. IEEE Trans. Antennas Propag. 45, 1467-1474 (1997)

4. KC Tan, KC Ho, A Nehorai, Linear independence of steering vectors of an electromagnetic vector sensor. IEEE Trans. Signal Process. 44, 3099-3107 (1996)

5. J Zhang, CC Ko, A Nehorai, Separation and tracking of multiple broadband sources with one electromagnetic vector sensor. IEEE Trans. Aerosp. Electron. Syst. 38, 1109-1116 (2002)

6. A Nehorai, P Tichavsky, Cross-product algorithms for source tracking using an EM vector sensor. IEEE Trans. Signal Process. 47, 2863-2867 (1999)

7. KT Wong, X Yuan, Vector cross-product direction-finding with an electromagnetic vector-sensor of six orthogonally oriented but spatially non-collocating dipoles/loops. IEEE Trans. Signal Process. 59, 160-171

(2011)

8. X Yuan, Estimating the DOA and the polarization of a polynomial-phase signal using a single polarized vector-sensor. IEEE Trans. Signal Process. 60, 1270-1282 (2012)

9. $\mathrm{BC}$ Ng, CMS See, Sensor array calibration using a maximum likelihood approach. IEEE Trans. Antennas Propag. 44, 827-835 (1996)

10. F Sellone, A Serra, A novel online mutual coupling compensation algorithm for uniform and linear arrays. IEEE Trans. Signal Process. $\mathbf{5 5}$ 560-573 (2007)

11. Z Ye, J Dai, XXu, X Wu, DOA estimation for uniform linear array with mutual coupling. IEEE Trans. Aerosp. Electron. Syst. 45, 280-288 (2009)

12. J Dai, Z Ye, Spatial smoothing for DOA estimation of coherent signals in the presence of unknown mutual coupling. IET Signal Process. 5, 418-425 (2011)

13. J Dai, W Xu, D Zhao, Real-valued DOA estimation for uniform linear array with unknown mutual coupling. Signal Process. 92, 2056-2065 (2012)

14. W Wang, S Ren, Y Ding, H Wang, An efficient algorithm for direction finding against unknown mutual coupling. Sensors. 14, 4-20077 (2006)

15. B Liao, S Chan, A cumulant-based approach for direction finding in the presence of mutual coupling. Signal Process. 104, 197-202 (2014)

16. J Dai, X Bao, N Hu, C Chang, W Xu, A Recursive RARE Algorithm for DOA Estimation With Unknown Mutual Coupling. IEEE Antennas Wirel. Propag. Lett. 13, 1593-1596 (2014)

17. M Lin, L Yang, Blind calibration and DOA estimation with uniform circular arrays in the presence of mutual coupling. IEEE Antennas Wirel. Propag. Lett. 5, 315-318 (2006)

18. J Dai, D Zhao, X Ji, A sparse representation method for DOA estimation with unknown mutual coupling. IEEE Antennas Wirel. Propag. Lett. 11 1210-1213 (2012)

19. $\mathrm{NHu}, \mathrm{Z} Y \mathrm{Y}, \mathrm{XXu}, \mathrm{M}$ Bao, DOA estimation for sparse array via sparse signal reconstruction. IEEE Trans. Aerosp. Electron. Syst. 49, 760-773 (2013)

20. J Dai, N Hu, W Xu, C Chang, Sparse Bayesian learning for DOA estimation with mutual coupling. Sensors. 15, 7-26280 (2626)

21. Y Wang, M Trinkle, BW-H Ng, DOA estimation under unknown mutual voupling and multipath with improved effective array aperture. Sensors. 15, 30856-30869 (2015)

22. G Wang, H Tao, J Su, X Guo, C Zeng, L Wang, in Antennas, Propagation \& EM Theory (ISAPE) 2012 10th International Symposium on. Mutual coupling calibration for electromagnetic vector sensor array (IEEE, Xian, 2012), pp. 261-264
23. L Wang, G Wang, C Zeng, Mutual coupling calibration for electromagnetic vector sensor. Prog. Electromagn. Res. B. 52, 347-362 (2013)

24. KC Ho, KC Tan, A Nehorai, Estimating directions of arrival of completely and incompletely polarized signals with electromagnetic vector sensors. IEEE Trans. Signal Process. 47, 2845-2852 (1999)

25. K Wang, J He, T Shu, Z Liu, Localization of mixed completely and partially polarized signals with crossed-dipole sensor arrays. Sensors. 15, 31859-31868 (2015)

26. AN Lemma, AJ van der Veen, EF Deprettere, Analysis of joint angle-frequency estimation Using ESPRIT. IEEE Trans. Signal Process. 51, 1264-1283 (2003)

27. MC Vanderveen, AJ van der Veen, A Paulraj, Estimation of multipath parameters in wireless communications. IEEE Trans. Signal Process. 46 682-690 (1998)

28. R Roy, T Kailath, ESPRIT-estimation of signal parameters via rotational invariance techniques. IEEE Trans. Acoust. Speech Signal Process. 37 984-995 (1989)

\section{Submit your manuscript to a SpringerOpen ${ }^{\circ}$ journal and benefit from:}

- Convenient online submission

- Rigorous peer review

- Open access: articles freely available online

- High visibility within the field

- Retaining the copyright to your article

Submit your next manuscript at $\$$ springeropen.com 